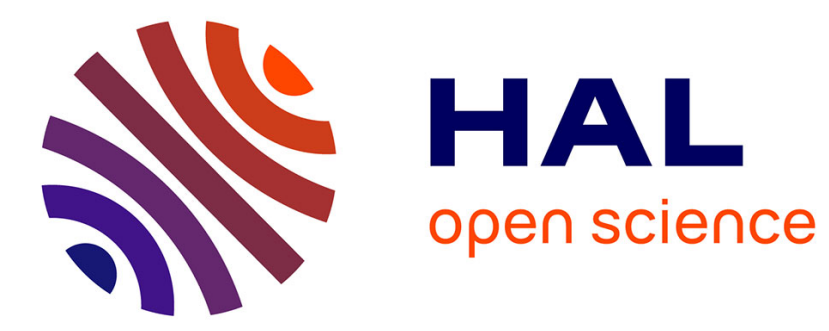

\title{
A continuum damage modeling of quasi-static fatigue strength of plain concrete
}

Si Hai Mai, Frédéric Le-Corre, Gilles Forêt, Boumediene Nedjar

\section{To cite this version:}

Si Hai Mai, Frédéric Le-Corre, Gilles Forêt, Boumediene Nedjar. A continuum damage modeling of quasi-static fatigue strength of plain concrete. International Journal of Fatigue, 2012, 37, pp.79-85. 10.1016/j.ijfatigue.2011.10.006 . hal-00668020

\section{HAL Id: hal-00668020 https://hal.science/hal-00668020}

Submitted on 9 Feb 2012

HAL is a multi-disciplinary open access archive for the deposit and dissemination of scientific research documents, whether they are published or not. The documents may come from teaching and research institutions in France or abroad, or from public or private research centers.
L'archive ouverte pluridisciplinaire HAL, est destinée au dépôt et à la diffusion de documents scientifiques de niveau recherche, publiés ou non, émanant des établissements d'enseignement et de recherche français ou étrangers, des laboratoires publics ou privés. 


\title{
A continuum damage modelling of quasi-static fatigue strength of plain concrete
}

\author{
S. H. Mai ${ }^{\mathrm{a}, \mathrm{b}}$, F. Le-Corre ${ }^{\mathrm{b}}$, G. Foret ${ }^{\mathrm{a}}$, B. Nedjar ${ }^{\mathrm{a}, *}$ \\ ${ }^{a}$ Université Paris-Est, Laboratoire Navier (ENPC/IFSTTAR/CNRS), \\ Ecole des Ponts ParisTech, 6 et 8 avenue Blaise Pascal, 77455 Marne-la-Vallée, France \\ ${ }^{b}$ RED Railway Track, Transport Global Solution (TGS), ALSTOM Transport, \\ 48 rue Albert Dhalenne, 93482 Saint-Ouen, France
}

\begin{abstract}
A three-dimensional phenomenological model is developed to describe the fatigue behaviour of concrete. The approach is based on the framework of continuum damage mechanics where the fatigue model is derived by means of Marigo's formulation where the yield concept is replaced by a loadingunloading irreversibility concept allowing to account for damage accumulation even for loading levels below the yield surface. Specifically, a static damage model is proposed and deeply investigated. Its extension to the fatigue range is proposed through two different functions of the power-lawtype. While the first one only needs one additional parameter, the second one needs three additional parameters. In all cases, these latters need to be identified by means of fatigue tests through Wöhler curves. In this paper, flexural fatigue tests have been performed on a concrete the formulation of which is close to the one used for urban railway slabs. A set of numerical examples is presented. It shows good agreements with the present experimental
\end{abstract}

\footnotetext{
*Corresponding author: boumediene.nedjar@enpc.fr, Tel. +33-1-64153715
} 
results together with some data found in the literature.

Keywords: Fatigue, Concrete, Wöhler curves, Flexion, Continuum Damage

\section{Introduction}

Track degradation is a very huge domain. It consists of many different problems such as wear, corrugation and fatigue cracks. For each problem, different mechanisms can occur separately or simultaneously and many parameters have to be taken into account. In this paper, attention is focused on concrete railway slabs which, under several thousands of load cycles, may experience fatigue degradation. It is then of interest to provide life-time predictive tools within a phenomenological modelling framework.

Fatigue performance of concrete has been studied for several decades by many authors. Under compression, see for example [1, 2, 3], and under flexural tests, see for example $[4,5,6,7]$ to mention few. The most apparent observation from the published experimental results is their marked diversity and dispersion. Moreover, experimental tests show different results even with samples made with the same concrete. For this, experimental campaigns need repeated tests to approach a mean behaviour of the material. This is mostly due to the random nature of the concrete material itself.

In this study, an experimental campaign has been made on a concrete with a formulation given in Table 1. This formulation has been chosen because it results to a concrete with mechanical properties close enough to the one used for urban railways' slabs. Three points bending tests have been performed on 33 plain concrete beams samples of equal dimensions $(50 \times 100 \times 400) \mathrm{mm}^{3}$. 18 samples have been submitted to monotonic loadings until fracture to measure 
the strength of each sample while the 15 remaining samples have been used for fatigue tests. Five levels of loading have been applied, each of which with three samples. All the experiments were conducted at the frequency range of $10 \mathrm{~Hz}$, i.e. with 10 loading cycles per second. The results are collected in Table 2 where the loading level $\mathrm{S}$ is the ratio between the amplitude of the applied cyclic force and the strength obtained from the monotonic tests. And where $\mathrm{N}$ is the number of cycles to failure. Notice that for the sample 3 loaded with the amplitude $\mathrm{S}=0.6$, the test has been stopped after $410^{7}$ cycles $(\approx 47$ days $)$.

Table 1: Concrete slab formulation. The quantities are given in $\left[\mathrm{kg} / \mathrm{m}^{3}\right][8]$.

\begin{tabular}{|l|l|l|l|l|}
\hline aggregates & sand & cement & superplasticizer & water \\
\hline 1055.1 & 819.9 & 350 & 3.5 & 169.6 \\
\hline
\end{tabular}

Table 2: S-N experimental results of flexural fatigue strength [8].

\begin{tabular}{|l|l|l|l|l|l|}
\hline & $\mathrm{S}=0.6$ & $\mathrm{~S}=0.71$ & $\mathrm{~S}=0.74$ & $\mathrm{~S}=0.8$ & $\mathrm{~S}=0.84$ \\
\hline $\mathrm{N}$ for sample 1 & $1.610^{6}$ & 118000 & 59000 & 20000 & 1000 \\
\hline $\mathrm{N}$ for sample 2 & $3.610^{6}$ & 122000 & 63000 & 25000 & 1250 \\
\hline $\mathrm{N}$ for sample 3 & $>410^{7}$ & 200000 & 100000 & 35000 & 3100 \\
\hline
\end{tabular}

In this paper, we choose to describe the material degradation by means of the nowadays classical framework of continuum damage mechanics. Few contributions in the literature have used this formulation for fatigue modelling, see for example $[9,3,10]$. It is based on the thermodynamics of irreversible 
processes where damage is described by means of an internal quantity. In the following, the stress-strain constitutive law we adopt constitutes the simplest model where damage is coupled to elasticity. We write

$$
\boldsymbol{\sigma}=(1-D) \mathbf{C}: \boldsymbol{\varepsilon}
$$

where $\boldsymbol{\sigma}$ and $\boldsymbol{\varepsilon}$ are the second order stress and strain tensors, respectively, $\mathbf{C}$ is the fourth-order undamaged Hooke's elasticity tensor, and where the scalar $D$ is the internal damage variable with value 0 when the material is undamaged and value 1 when the material is completely damaged. In (1) and in all what follows, the double dot symbol : denotes the double tensor contraction. It is to be stressed that with the choice (1), we exclude the inelastic strains which would probably involve interactions with damage within a more elaborated modelling framework. Moreover, and as quasi-statics is of interest in this work, we a priori exclude the eventual time-dependent dynamic effects.

The way the internal variable $D$ evolves strongly depends on the damage flow rule. The fatigue damage model adopted in this paper follows the relaxation formalism as proposed by Marigo [11], and is constructed from a static local damage model. This latter is written in classical form and is first introduced in Section 2.

The rest of the paper is organized as follows. In Section 2, the static damage model is deeply developed and discussed before its extension to its fatigue damage version in Section 3. Aspects on its numerical integration within the finite element method are detailed in Section 4. Then, a set of numerical examples are compared to the exprimental test results mentioned above in Section 5, together with other results found in the literature. And finally, conclusions are drawn in Section 6. 


\section{Static damage modelling framework}

By static damage modelling we mean mechanical damage response under monotonic loading. Moreover, in this paper, cyclic loading with increasing amplitude also falls within the scope of this terminology. The idea underlining the continuum damage model presented in this work is the hypothesis that damage is directly linked to the strain history. In this section, we develop a local damage model by means of the nowadays well known yield concept. It is firstly detailed and discussed in its classical form. Then, for later use in fatigue, it is equivalently written in a form invoquing the gauge function.

\subsection{A strain-based damage model}

To characterize the progressive degradation of the mechanical properties, we choose a damage criterion formulated in strain space as

$$
g(D ; \boldsymbol{\varepsilon})=(1-D)^{m} S(\boldsymbol{\varepsilon})-W \leq 0
$$

where $W$ is the initial damage threshold and $S(\varepsilon)$ is the driving source of damage which depends solely on the strain $\varepsilon$. $W$ and $S$ are expressed in terms of volumetric energies $\left[\mathrm{Nm} / \mathrm{m}^{3}\right]$. And the up to now constant dimensionless exponent parameter $m>0$ controls the hardening or the softening material response as will be discussed bellow. We next define the evolution of the damage variable $D$ by the rate equation

$$
\dot{D}=\delta
$$


where $\delta$ is a damage consistency parameter that defines damage loading/unloading conditions according to the Kuh-Tucker relations

$$
\delta \geq 0, \quad g(D ; \varepsilon) \leq 0, \quad \delta g(D ; \varepsilon)=0 .
$$

This model is no more than the local version of the gradient damage model presented in $[12,13]$. If $g(D ; \varepsilon)<0$, the damage criterion is not satisfied and condition $(4)_{3}$ gives $\delta=0$, hence the damage rule (3) implies that $\dot{D}=0$ and no further damage takes place. If, on the other hand, $\delta>0$; that is further damage is taking place, $(4)_{3}$ now implies $g(D ; \varepsilon)=0$. In this event the value of $\delta$ is determined by the damage consistency condition $\dot{g}(D ; \varepsilon)=0$ which gives in our case

$$
\delta \equiv \dot{D}=\frac{1-D}{m S(\varepsilon)} \dot{S}(\varepsilon)
$$

This latter has a form that matches the general framework proposed in [14]. Now to gain further insight into the nature of the present damage model, let us express in rate form the one-dimensional version of (1), i.e. $\sigma=(1-D) E \varepsilon$ where $E$ is the Young's modulus. And, moreover, let the driving source of damage be given for simplicity by the effective strain energy: $S(\varepsilon)=1 / 2 E \varepsilon^{2}$ which implies $\dot{S}(\varepsilon)=E \varepsilon \dot{\varepsilon}$, both these latter quantities to be replaced in the expression (5). One obtains then

$$
\dot{\sigma}=(1-D) E \dot{\varepsilon}-\dot{D} E \varepsilon \equiv(1-D)\left\{1-\frac{2}{m}\right\} E \dot{\varepsilon} .
$$

It is a well known theoretical result that the tangent modulus is positive for hardening material response, and is negative in the case of softening. Then, as $(1-D) \geq 0$, the sign of the tangent modulus deduced from the rate form (6) depends solely on the value of the exponent parameter $m$. We then 
conclude that, in the present form, the elastic-damage model exhibits pure hardening for $m>2$, or pure softening for $m<2$.

However, for most concrete materials, the behaviour in compression is characterized by a hardening response when damage initiates until a pic load is reached, and then followed by a material softening until complete degradation. Hence, to take into account those facts, the exponent material parameter $m$ should not be kept constant but must be variable. In this work, we choose to make it damage-dependent through the following form

$$
m(D)=m_{1}(1-D)^{m_{2}}+m_{3}
$$

with $m_{1} \geq 0, m_{2}>0$ and $m_{3}>0$ being from now new material parameters. That is, when damage initiates $(D \approx 0)$, one has $m \approx m_{1}+m_{3}$, and $m$ tends to $m_{3}$ when $D$ approaches complete degradation $(D \approx 1)$. Hence, for instance, choosing the parameters $m_{1}$ and $m_{3}$ such that $m_{1}+m_{3}>2$ with $m_{3}<2$ allows to cover both the hardening and softening stages of the material response. The convenient parameter $m_{2}$ controls the rate at which the transition from hardening to softening is achieved. Also, notice that for $m_{1}=0$, the original model with constant exponent $\left(m \equiv m_{3}\right)$ is reached.

Last but not least, as it is also observed that damage within concrete is mostly produced by extensions, we choose the driving source of damage $S(\varepsilon)$ to depend on positive strain quantities through the following volumetric enery form

$$
S(\varepsilon)=\frac{1}{2}\left\{2 \mu \varepsilon^{+}: \varepsilon^{+}+\lambda\left(\langle\operatorname{tr}[\varepsilon]\rangle^{+}\right)^{2}\right\}
$$

where $\lambda$ and $\mu$ are the Lamé coefficients, $\operatorname{tr}[$.$] designates the trace oper-$ ator of second-order tensors, $\langle.\rangle^{+}$is the positive part scalar function as 
$\langle x\rangle^{+}=\frac{1}{2}\{x+|x|\}$, and where the positive part $\varepsilon^{+}$of the strain tensor $\varepsilon$ is obtained after diagonalisation. In fact, as this latter is symmetric, its spectral decomposition is given as

$$
\boldsymbol{\varepsilon}=\sum_{A=1}^{3} \varepsilon_{A} \boldsymbol{n}^{(A)} \otimes \boldsymbol{n}^{(A)},
$$

where $\left\{\varepsilon_{A}\right\}_{A=1,2,3}$ are the principal strains with $\left\{\boldsymbol{n}^{(A)}\right\}_{A=1,2,3}$ being the corresponding principal directions. In (9), the symbol $\otimes$ designates the tensor product. Hence, $\boldsymbol{\varepsilon}^{+}$is then given as

$$
\boldsymbol{\varepsilon}^{+}=\sum_{A=1}^{3}\left\langle\varepsilon_{A}\right\rangle^{+} \boldsymbol{n}^{(A)} \otimes \boldsymbol{n}^{(A)},
$$

and the source of damage function in (8) is equivalently written as

$$
S(\varepsilon)=\mu\left\{\left(\left\langle\varepsilon_{1}\right\rangle^{+}\right)^{2}+\left(\left\langle\varepsilon_{2}\right\rangle^{+}\right)^{2}+\left(\left\langle\varepsilon_{3}\right\rangle^{+}\right)^{2}\right\}+\frac{1}{2} \lambda\left(\left\langle\varepsilon_{1}+\varepsilon_{2}+\varepsilon_{3}\right\rangle^{+}\right)^{2} .
$$

In summary: the static damage model in its classical form is given by the constitutive equation (1), the damage criterion (2) with $m \equiv m(D)$ and $S(\varepsilon)$ given by the expressions (7) and (11), respectively, and by the damage flow given by the constrained damage flow problem (3)-(4).

\subsection{Equivalent formulation of the static damage model}

The damage criterion (2) can be alternatively written in an equivalent form as

$$
0 \leq f(D ; \varepsilon)=\frac{(1-D)^{m(D)} S(\varepsilon)}{W} \leq 1
$$

where the left inequality holds since $W$ is a positive material parameter, $S(\varepsilon)$ is a positive energy norm and the internal damage variable $D$ belongs to the 
interval $[0,1]$. Here and in all what follows, the above function $f$ is defined as the gauge function characterizing the damage yield criterion.

When further damage takes place, the damage consistency condition $\dot{g}(D ; \varepsilon)=0$ is then equivalently written as $\dot{f}(D ; \varepsilon)=0$ which gives

$$
\dot{D}=-\frac{1}{\frac{\partial f}{\partial D}} \frac{\partial f}{\partial \varepsilon}: \dot{\varepsilon} .
$$

Then, replacing $f$ by its expression in (12), the constrained damage flow problem (3)-(4) is equivalently written as

$$
\dot{D}= \begin{cases}0 & \text { if } f<1, \\ \frac{1}{\frac{m(D)}{1-D}-m^{\prime}(D) \ln (1-D)}\left\langle\frac{\partial S(\boldsymbol{\varepsilon})}{\partial \boldsymbol{\varepsilon}}: \dot{\boldsymbol{\varepsilon}}\right\rangle^{+} & \text {if } f=1,\end{cases}
$$

where the prime notation $m^{\prime}(D)$ designates the derivative of the function $m(D)$ with respect to its argument $D$ (see equation (7)), and where $\ln ($. is the natural logarithm function. Notice that if $m(D)$ is constant, $(14)_{2}$ reduces exactly to the expression (5).

One can observe that for a state $(D, \boldsymbol{\varepsilon})$ on the yield surface, i.e. with $f(D ; \boldsymbol{\varepsilon})=1$, the real scalar quantity $[\partial S(\varepsilon) / \partial \boldsymbol{\varepsilon}]: \dot{\boldsymbol{\varepsilon}}$ drives the damage flow and, hence, can be interpreted as a driving loading/unloading criterion.

\section{The fatigue damage model}

From the physical point of view, the fatigue material response is characterized by the accumulation of damage under repeated loadings even with levels below the damage yield surface, i.e. for $f<1$ (or $g<0$ ). The static 
damage model developed in Section 2 is unable to predict such a behaviour since no damage increase takes place if after unloading and subsequent reloading the strain does not exceed the maximum previously experienced value. Hence, this model should be extended to fatigue.

Marigo's formulation of fatigue [11] constitutes a sound way to make this extension. The key idea is to modify the original model by replacing the yield concept with an alternative loading/unloading irreversibility concept allowing damage evolution even for loading states below the yield surface. It is to be stressed that Marigo's formulation is general enough and can be applied to almost any local static damage model based on a yield concept, i.e. a model with a damage criterion and a constrained damage flow rule.

In our case, starting from the equivalent static damage formulation invoking the gauge function of Section 2.2, the constrained damage flow rule (14) is replaced by the following unconstrained evolution equation

$$
\dot{D}=\frac{\frac{1}{S(\varepsilon)}}{\frac{m(D)}{1-D}-m^{\prime}(D) \ln (1-D)} h(f)\left\langle\frac{\partial S(\varepsilon)}{\partial \varepsilon}: \dot{\varepsilon}\right\rangle^{+},
$$

where the newly introduced function $h(f)$ is any continuous and increasing function such that $h(0)=0$ and $h(1)=1$. It is evaluated at $f$ which, in turn, is the value of the gauge function $(12)$ at the current state $(D, \varepsilon)$. Focusing on this equation, three observations must be pointed out:

- Damage evolution can take place even for states below the yield surface with $f(D ; \varepsilon)<1$ (or equivalently with $g(D ; \varepsilon)<0$ ). In fact the function $h(f)$ in (15) equals zero only for $f=0$.

- The above highlighted scalar quantity $[\partial S(\varepsilon) / \partial \boldsymbol{\varepsilon}]: \dot{\varepsilon}$ plays the role of 
the loading/unloading irreversibility concept. In fact, Damage evolves if this latter is strictly positive, whereas no further damage takes place if it is negative.

- In the limiting case where the function $h$ is replaced by the Heaviside function $H(f-1)$ such as $h(f)=0$ if $f \leq 1$ and $h(f)=1$ if $f \geq 1$, the evolution equation (15) tends to the original constrained static damage flow rule (14).

Within the few contributions in the literature that used this formulation, see for instance $[9,3,10]$, a power law has been chosen for the function $h$

$$
h(f)=f^{n},
$$

where the exponent $n>0$ is the lonely additional material parameter, besides on the static damage model parameters. This choice will also be adopted in this paper.

Another alternative choice will also be used in this work

$$
h(f)= \begin{cases}\alpha^{n_{1}}\left(\frac{f}{\alpha}\right)^{n_{2}} & \text { if } 0 \leq f \leq \alpha, \\ f^{n_{1}} & \text { if } \alpha \leq f \leq 1\end{cases}
$$

where, this time, three new material parameters are added: the exponents $n_{1}>0, n_{2}>0$ and the parameter $\alpha$ such that $\left.\left.\alpha \in\right] 0,1\right]$. Notice that for $\alpha=1$, the function (17) reaches the power law with $n=n_{2}$, and for $\alpha \rightarrow 0$ it tends to the power law with $n=n_{1}$. Notice also that this function matches the required conditions mentioned above especially the continuity at $f=\alpha$. The parameter $\alpha$ is in fact a transition point between two power laws. Hence, 
for later use in this paper, the function (17) will be refered to as the bi-power law.

In summary: the fatigue damage model is given by the constitutive equation (1), the gauge function $f$ defined in (12) and the damage evolution equation (15) where the function $h(f)$ is defined by either (16) or (17).

\section{Numerical integration of the fatigue law}

In this section, we consider an algorithmic approximation consistent with the fatigue damage model developed above. In a finite element context, this is accomplished at the integration point level through a strain-driven numerical procedure. Consider a typical time interval $\left[t_{n}, t_{n+1}\right]$, an arbitrary material point $\boldsymbol{x}$ and assume that the variables $\left(\varepsilon_{n}, D_{n}\right)$ are known prescribed initial data on $\boldsymbol{x}$ at time $t_{n}$. The objective is then to approximate the evolution equation to advance the solution to time $t_{n+1}$ and update the variables to $\left(\varepsilon_{n+1}, D_{n+1}\right)$ for a fixed increment of deformation.

\subsection{An implicit-like numerical scheme}

Within a standard displacement finite element method, the nodal displacements are updated iteratively at the global level and, hence, the incremented strain tensor $\varepsilon_{n+1}$ is known during the local iterative procedure at the integration point level. Two situations can occur:

- if $\varepsilon_{n+1} \leq \varepsilon_{n}$, then the criterion is negative and no further damage takes place. We then set $D_{n+1}=D_{n}$.

- else if $\varepsilon_{n+1}>\varepsilon_{n}$, then an incrementally damage flow process is taking place. 
In the case of damage growth, the evolution equation (15) is approximated by the following implicit-like finite difference scheme

$$
D_{n+1}-D_{n}=\frac{1}{\frac{m_{n+1}}{1-D_{n+1}}-m_{n+1}^{\prime} \ln \left(1-D_{n+1}\right)} \zeta_{n+1}
$$

with the short hand notation $\zeta_{n+1}$ given by

$$
\zeta_{n+1}=\frac{\tilde{h}_{n+1}}{S_{n+1}}\left[\frac{\partial S}{\partial \varepsilon}\right]_{n+1}:\left(\varepsilon_{n+1}-\varepsilon_{n}\right),
$$

where $m_{n+1} \equiv m\left(D_{n+1}\right), m_{n+1}^{\prime} \equiv m^{\prime}\left(D_{n+1}\right), S_{n+1} \equiv S\left(\varepsilon_{n+1}\right)$ and $\tilde{h}_{n+1} \equiv$ $h\left(f\left(D_{n} ; \boldsymbol{\varepsilon}_{n+1}\right)\right)$. This latter means that the function $h$ (either given by (16) or $(17))$ is evaluated at a mixed state $\left(D_{n}, \boldsymbol{\varepsilon}_{n+1}\right)$. This choice is justified by the fact that damage evolves slowly within the increments of a single cycle with regards to the whole fatigue process. Hence, $\zeta_{n+1}$ is obtained by mere function evaluation and is considered fixed at this level. Details about the derivative term $\partial S / \partial \boldsymbol{\varepsilon}$ will be given in the next subsection.

The nonlinear equation (18) can be solved locally for $D_{n+1}$ by means of a Newton iterative method. The details are ommited in this paper since this procedure is simple.

\subsection{The continuum tangent moduli}

The initial boundary value problem of fatigue is nonlinear. The source of nonlinearity is a material one which arises from the constitutive relation. Hence, this problem is solved at the global level by means of an iterative process of the Newton's type. Accordingly, this requires the linearization of the equilibrium problem about a known state at time $t_{n}$. This procedure is nowadays standard. We give here the contribution to the tangent stiffness 
where it is of interest to determine the relation between the rate of stress and the rate of strain via the change of the internal damage variables $D$.

Starting from the rate form of the constitutive relation (1) one obtains

$$
\dot{\boldsymbol{\sigma}}=(1-D) \mathbf{C}: \dot{\boldsymbol{\varepsilon}}-\dot{D} \boldsymbol{\sigma}_{0}
$$

where $\boldsymbol{\sigma}_{0}=\mathbf{C}: \boldsymbol{\varepsilon}$ is the effective stress tensor. If no further damage takes place, the tangent moduli are simply given by $\mathbf{C}_{T}=(1-D) \mathbf{C}$, while in the case of damage growth, the evolution equation (15) replaced into (20) gives

$$
\mathbf{C}_{T}=(1-D) \mathbf{C}-\frac{h(f)}{\alpha(D) S(\varepsilon)} \boldsymbol{\sigma}_{0} \otimes \frac{\partial S(\boldsymbol{\varepsilon})}{\partial \boldsymbol{\varepsilon}}
$$

such that $\dot{\boldsymbol{\sigma}}=\mathbf{C}_{T}: \dot{\boldsymbol{\varepsilon}}$, and where we have used the short hand notation $\alpha(D)=m(D) /(1-D)-m^{\prime}(D) \ln (1-D)$.

Now it only remains to precise the expression of the symmetric secondorder tensor $\partial S / \partial \varepsilon$. As the source of damage function given by (11) is of the form $S=S\left(\varepsilon_{1}, \varepsilon_{2}, \varepsilon_{3}\right)$, by the chain rule together with the spectral decomposition (9), one obtains

$$
\frac{\partial S}{\partial \boldsymbol{\varepsilon}}=\sum_{A=1}^{3} \frac{\partial S}{\partial \varepsilon_{A}} \boldsymbol{n}^{(A)} \otimes \boldsymbol{n}^{(A)},
$$

where the important spectral property

$$
\frac{\partial \varepsilon_{A}}{\partial \boldsymbol{\varepsilon}}=\boldsymbol{n}^{(A)} \otimes \boldsymbol{n}^{(A)}, \quad A=1,2,3
$$

has been used. And finally, with the other useful property relative to the positive part function that

$$
\frac{\partial\langle x\rangle^{+}}{\partial x}\langle x\rangle^{+}=\langle x\rangle^{+}
$$

for any value of the scalar $x$, we end up with the following expression

$$
\frac{\partial S}{\partial \varepsilon_{A}}=2 \mu\left\langle\varepsilon_{A}\right\rangle^{+}+\lambda\left\langle\varepsilon_{1}+\varepsilon_{2}+\varepsilon_{3}\right\rangle^{+}, \quad A=1,2,3
$$




\section{Numerical simulations of fatigue}

In this section, numerical simulations are compared to the experimental data from the present experimental compaign. Moreover, other simulated traction and compression fatigue strength tests are also compared to existing experimental results found in the literature. Both kind of results are given in the form of $\mathrm{S}-\mathrm{N}$ Wöhler curves. Let us stress that each point of these simulated curves corresponds to a cyclic computation for a given load amplitude where, within each cycle, the load is in turn discretized and incremented.

The mean values of the mechanical characteristics of the concrete formulated as in Table 1 are given in Table 3. A value $\nu=0.2$ has been used for the Poisson's ratio in the following computations.

Table 3: Mechanical characteristics of the concrete.

\begin{tabular}{|l|l|l|}
\hline Young's modulus & strength in traction & strength in compression \\
\hline $36.3 \mathrm{MPa}$ & $3.2 \mathrm{MPa}$ & $37 \mathrm{MPa}$ \\
\hline
\end{tabular}

In general, to identify the parameters of the fatigue damage model at hand from laboratory tests we proceed in two steps:

- In the first step, we capture the static damage behaviour of the material under monotonic or low cyclic loading with increasing amplitude. This permits to identify the elastic and the damage parts of the material parameters as developed in Section 2: the Young's modulus E, the Poisson's ratio $\nu$, the initial damage threshold $W$ and the parameters 
$m_{1}, m_{2}$ and $m_{3}$ of the exponent function given by (7). In particular, from the expression (2) (or (12)) of the damage criterion, the initial damage threshold $W$ corresponds to the source of damage function (11) evaluated at the strain state at which the stress-strain nonlinearity starts, i.e. at which damage initiates from our elastic-damage modelling point of view. Then, for instance, curve fitting can be used to identify the $m_{1}, m_{2}, m_{3}$ parameters from the nonlinear part of the response curves.

- And, in the second step, all what remains is to determine the additional fatigue parameter(s) as developed in Section 3: the parameter $n$ if the power law (16) is used, or the three parameters $n_{1}, n_{2}$ and $\alpha$ if the bi-power law (17) is chosen instead. Curve fitting can also be used to capture these parameters from S-N Wöhler curves.

\subsection{Flexural fatigue strength}

In multiaxial three points bending tests, it is mainly observed that concrete is more sensitive to traction than compression. Damage initiates and grows from the most stretched fibres until fracture. For this, it is customary to assume flexural tests as indirect traction tests for plain concrete. The behaviour we use in the following computations is given by the simulated curve illustrated in Figure 1 where the uniaxial static strength $R_{t}$ in traction is 3.202 MPa (compare with the experimental result of Table 3). The material parameters used to obtain this static damage stress-strain relation are

$$
\begin{aligned}
& E=36.3 M P a, \quad \nu=0.2 \\
& W=1.3310^{-4} M P a, \quad m_{1}=0, \quad m_{2}=0, \quad m_{3}=0.667,
\end{aligned}
$$


where the exponent function in (7) has been chosen to be constant with $m=m_{3}<2$, i.e. a pure softening response. This gives the characteristic shape of concrete response in tension where the behaviour is almost linear until the pic-load followed by a more or less sharp softening.

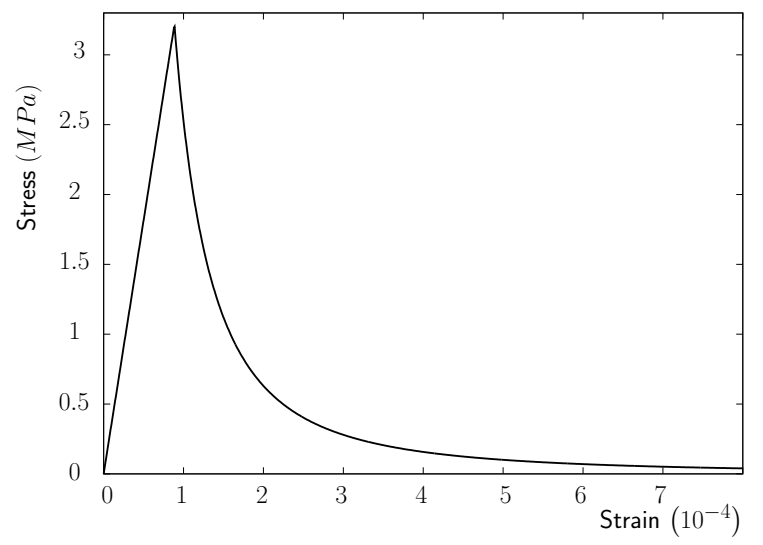

Figure 1: Stress-strain curve in uniaxial traction.

The response of the model is applied to the simulation of fatigue strength tests in traction by using either the power law (16) and the bi-power law (17). In Figure 2, the Wöhler curves of three computations are superposed to the experimental flexural fatigue results of Table 2: two computations using the power law with the parameter $n=15$ and $n=20$, and one computation using the bi-power law with the parameters $n_{1}=24, n_{2}=7$ and $\alpha=0.625$. Here the ordinate $S$ is the ratio between the stress load amplitude in traction and the simulated static strength in traction $R_{t}=3.202 \mathrm{MPa}$ (see Figure 1).

One can observe that the increase in cyclic amplitude leading to a decrease in fatigue life is well represented by Marigo's formulation of fatigue. And, as already mentioned in the literature where use has been made of the power 
law function (16), the number of cycles to failure increases with increasing $n$, see for instance $[3,10]$. Moreover, two further observations can also be pointed out:

- Having in mind that there are always dispersions in the experimental results, the power law function (16) can sometimes hardly fit these data as it depends on only one additional parameter. In Figure 2, for instance with $n=20$, the fatigue model under-estimates the strength in traction for $S=0.84$ and 0.8 , while it over-estimates it for $S=0.74,0.71$ and 0.6. And with $n=15$, the model under-estimates the strength until $\mathrm{S}=0.6$. This is confirmed for other values of the parameter $n$.

- Hence, the bi-power law (17) can be a good alternative to adjust the numerical results as it depends on more parameters. This can be noticed from the bi-power law curve shown in Figure 2 which better matches the experimental data.

For illustrative purposes, we have plotted in Figure 3 the maximum strain evolution for four selected computations that led to four simulated points on the bi-power law Wöhler curve of Figure 2 with the stress load amplitudes in traction: $\mathbf{S}=0.84,0.8,0.74$ and 0.71 . Also in Figure 3, we have plotted the intervals at which the flexural fatigue experimental tests led to failure for the same loading amplitudes. The ordinate at which these intervals are placed in the figure has no importance. Only the number of cycles is of interest. One can observe that, regardless of the cyclic amplitude, and also of the fatigue parameters, the curves have the same shape with two stages: a first stationary pseudo-linear cyclic creep phase followed by a fast secondary 


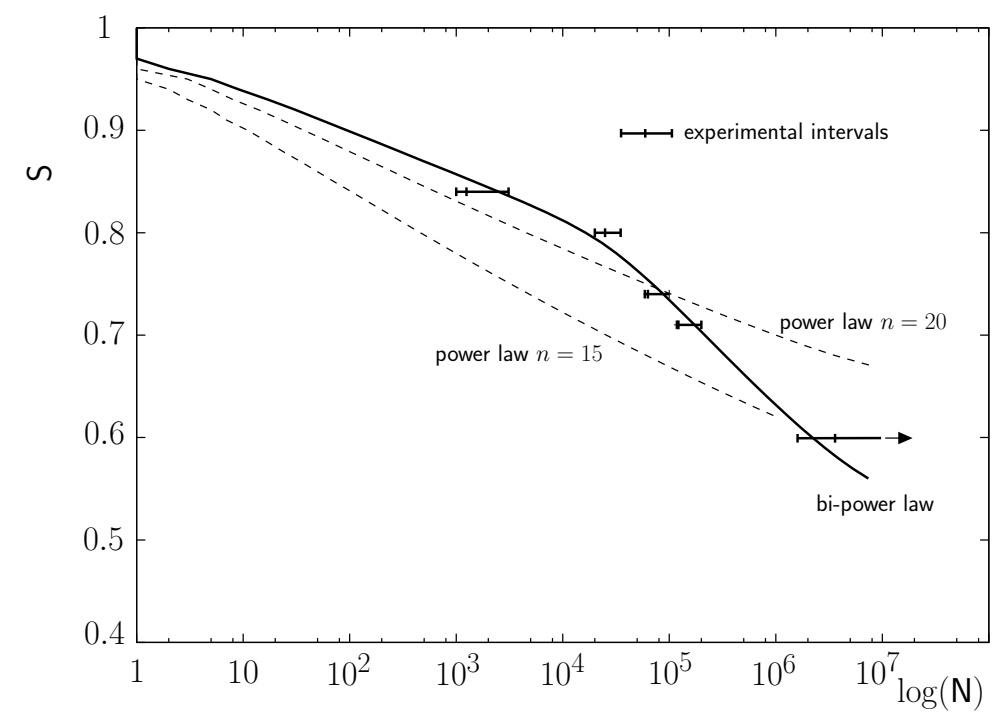

Figure 2: Simulated "S - N" Wöhler curves superposed to the experimental flexural fatigue results: two curves using the power law with $n=15$ and $n=20$, and one curve using the bi-power law with $n_{1}=24, n_{2}=7$ and $\alpha=0.625$. 
cyclic creep phase before failure.

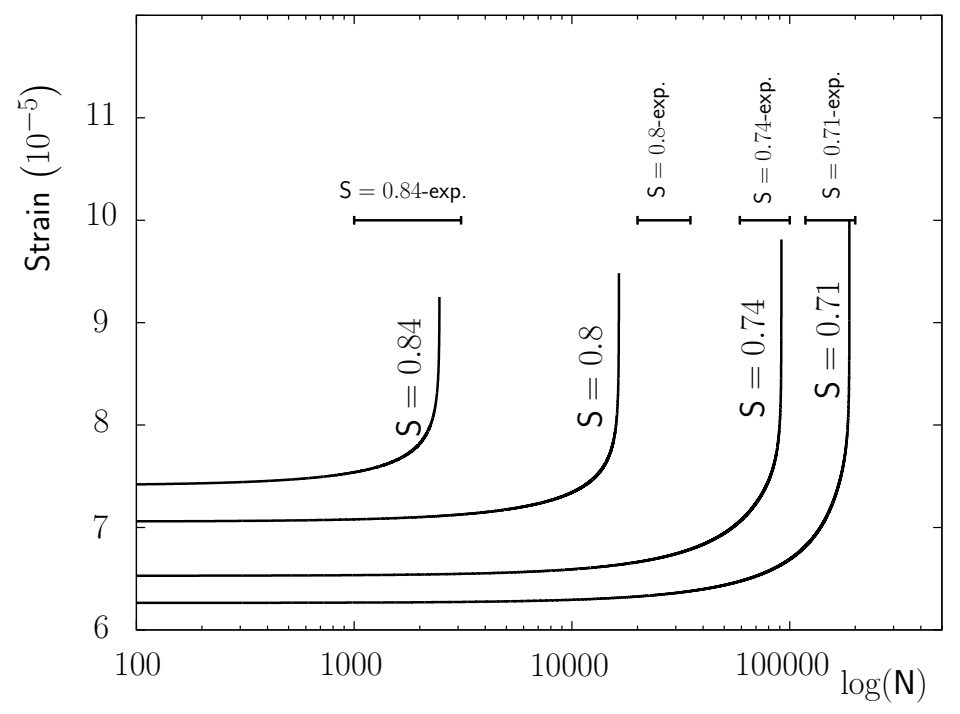

Figure 3: Cyclic creep. Model response of maximum strain evolution in traction fatigue using the bi-power law with $n_{1}=24, n_{2}=7$ and $\alpha=0.625$. Comparison with the number of experimental cycles obtained during the flexural tests.

Last but not least, the intrinsic material parameters, and especially in the present case, the fatigue material parameters greatly depend on the type of concrete under study. As an example, Figure 4 shows the simulated fatigue strength in traction of a concrete given in [7] with the model developed in this paper where use has been made of the following fatigue parameters of the bi-power law function (17)

$$
n_{1}=26, \quad n_{2}=11, \quad \alpha=0.85,
$$

and where, for the sake of simplicity, the same static stress-strain behaviour as for the precedent simulations has been used (see Figure 1), i.e. with the material parameters (26) for the static damage behaviour. 


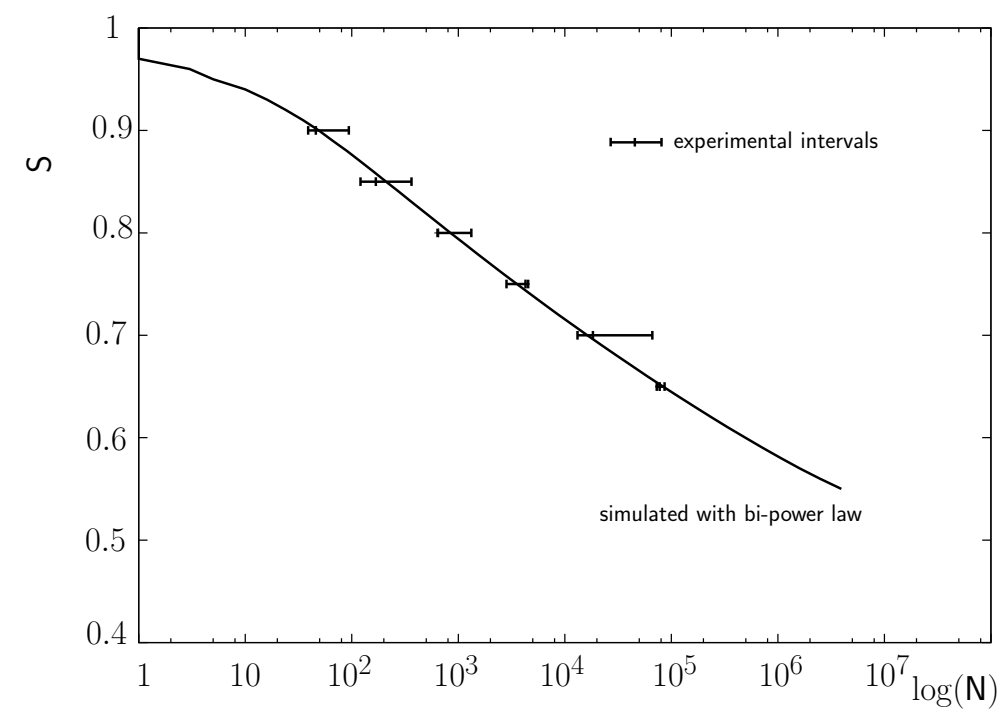

Figure 4: Simulated Wöhler curve from experimental traction fatigue tests after [7]. The bi-power law has been used with $n_{1}=26, n_{2}=11$ and $\alpha=0.85$.

\subsection{Simulations of fatigue strength in compression}

To get further insight into the modelling framework developed in this paper, we give in this section a numerical example of compressive fatigue strength. We first capture the static damage behaviour postulating a ductile response. The simulated stress-strain relation we use in the following computations is given in Figure 5 with the following material parameters

$$
\begin{aligned}
& E=36.3 M P a, \quad \nu=0.2 \\
& W=1.3310^{-4} M P a, \quad m_{1}=6, \quad m_{2}=0.7, \quad m_{3}=1.3
\end{aligned}
$$

The resulting uniaxial static strength in compression is $R_{c}=37.75 \mathrm{MPa}$ (compare with the experimental result of Table 3). Two further important observations must be pointed out:

- To obtain the above strength $R_{c}$, the set of parameters (28) is not 


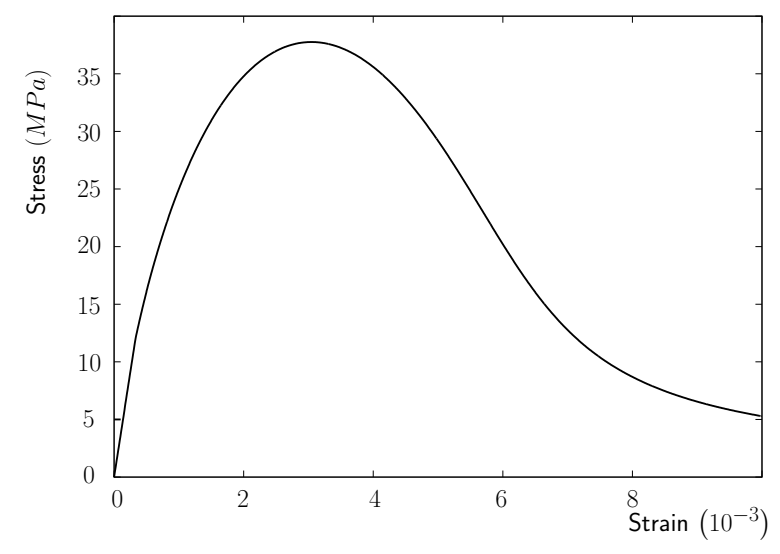

Figure 5: Simulated stress-strain behaviour in uniaxial compression.

unique. For instance, with a different initial damage threshold $W$ and different parameters $m_{1}, m_{2}, m_{3}$ than those given in (28), on can obtain the same strength, but at a different strain.

- If, for example, the compressive load direction coincides with the global $\vec{e}_{1}$-axis, the principal strain $\varepsilon_{1}$ is then negative while the other two principal strains are equal and positive due to the Poisson's ratio effect. Then, as the volumetric strain is negative, only the first term of the driving source of damage function $S(\varepsilon)$ in (11) (or (8)) contributes to the damage evolution.

In Figure 6, a fatigue compression response has been simulated and compared to the experimental results on a high strength concrete found in reference [2]. The bi-power law function (17) has been used with the parameters

$$
n_{1}=22, \quad n_{2}=10, \quad \alpha=0.9
$$

Here the ordinate $\mathbf{S}$ is the ratio between the stress load amplitude in 
compression and the simulated static strength $R_{c}$. One can notice the good quantitative agreement with the experimental data.

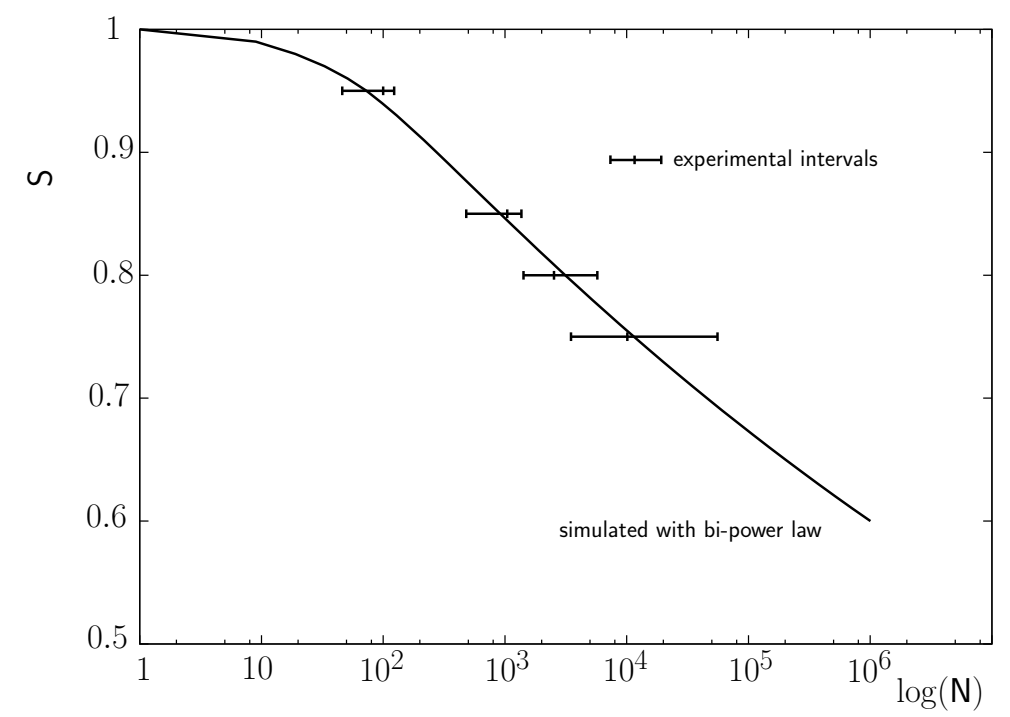

Figure 6: Simulated Wöhler curve from experimental fatigue test results in compression (after [2]).

Again for illustrative purposes, in Figure 7, we have selected the four computations that led to the four points of the Wöhler curve of Figure 6 for the loading amplitudes $\mathrm{S}=0.9,0.85,0.8$ and 0.75 .

Regardless of the cyclic amplitude, one can observe that the curves have the typical S-shape so that three stages can be noticed: a first very fast transient creep phase, a second stationnary pseudo-linear cyclic creep phase, and a fast tertiary cyclic creep phase before failure. 


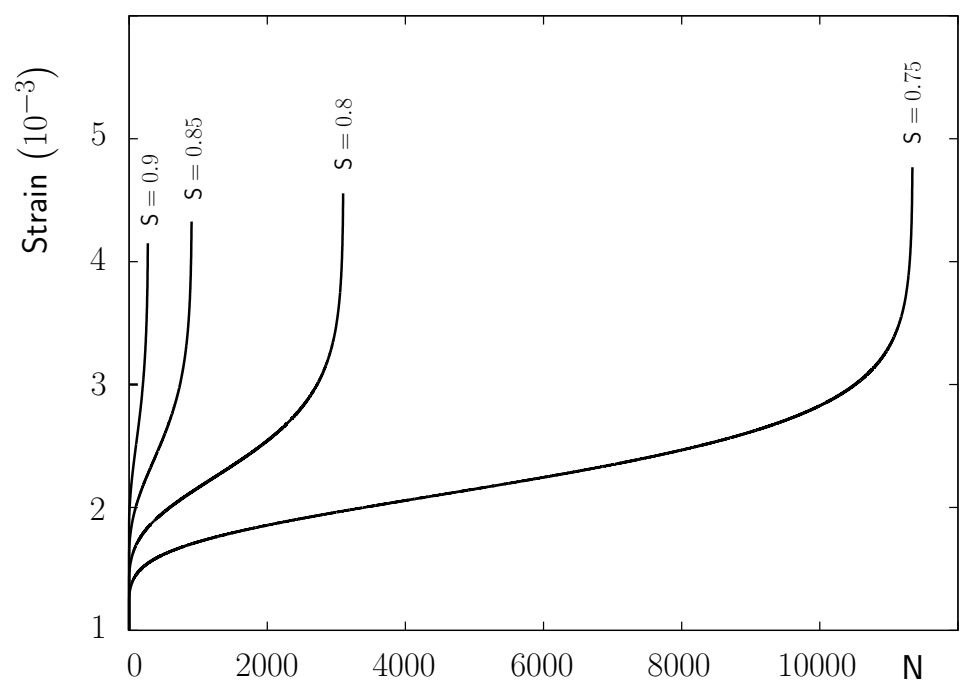

Figure 7: Cyclic creep. Model response of maximum strain evolution in compressive fatigue for the amplitudes $\mathrm{S}=0.9,0.85,0.8$ and 0.75 . The bi-power law function is used with $n_{1}=22, n_{2}=10$ and $\alpha=0.9$.

\section{Conclusions}

The main thrust of this paper has been the pesentation of a model in order to provide a tool for structural simulations under fatigue. The model developed is based on the nowadays well know continuum damage mechanics framework.

As it is mainly observed that the static mechanical response differs between tension and compression, we have first developed a damage model in a local classical form by means of a yield function. It permits to consider either the sharp sofening behaviour in tension and the ductile behaviour in compression. Then, by means of the deduced gauge function and by replacing the notion of yield surface by a relaxed loading/unloading irreversibility concept, we have shown that Marigo's fatigue formulation offers an ideal framework 
where one can take into account the damage accumulation even for loading amplitudes below the damage yield surface. Two alternatives have been proposed for the fatigue damage evolution: a classical power law function, and a bi-power law function introduced in this paper. This latter has shown to give better matching with the experimental results as it depends on more parameters than the power law function. And for both cases, a detailed algorithmic treatment has been developed to numerically solve the nonlinear evolution equations at hand.

It has been shown through a set of numerical simulations that the present fatigue damage model is able to capture the fatigue behaviour in tension and compression. A good accordance with the data obtained from the present experimental compaign, and with selected experimental results found in the literature has been obtained.

\section{References}

[1] Do MT, Chaallal O, Aitcin PC. Fatigue behavior of high-performance concrete. Journal of Materials in Civil Engineering 1993;15(1):96-111.

[2] Kim JK, Kim YY. Experimental study of the fatigue behaviour of high strength concrete. Cement and Concrete Research 1996;26(10):1513-23.

[3] Papa E. A damage model for concrete subjected to fatigue. European Journal of Mechanics, A/Solids 1993;12(3):429-40.

[4] Johnston CD, Zemp RW. Flexural fatigue performance of steel fiber reinforced concrete- Influence of fiber content, aspect ratio, and type. ACI Materials Journal 1991;88(4):374-83. 
[5] Oh BH. Fatigue life distributions of concrete for various stress levels. ACI Materials Journal 1991;88(2):122-8.

[6] Shi XP, Fwa TF, Tan SA. Flexural fatigue strength of plain concrete. ACI Materials Journal 1993;90(5):435-40.

[7] Zhang B, Phillips DV, Wu K. Further research on fatigue properties of plain concrete. Magazine of Concrete Research 1997;49(180):241-52.

[8] Mai SH. Etude de dégradation des voies ferrées urbaines (in french). Ph.D. thesis; Université Paris-Est; 2011.

[9] Alliche A. Damage model for fatigue loading of concrete. International Journal of Fatigue 2004;26:915-21.

[10] Papa E, Taliercio A. Anisotropic damage model for the multiaxial static and fatigue behaviour of plain concrete. Engineering Fracture Mechanics 1996;55(2):163-79.

[11] Marigo JJ. Modelling of brittle and fatigue damage for elastic material by growth of microvoids. Engineering Fracture Mechanics 1985;21(4):861-74.

[12] Nedjar B. Elastoplastic-damage modelling including the gradient of damage. Formulation and computational aspects. International Journal of Solids and Structures 2001;38:5421-51.

[13] Nedjar B. A theoretical and computational setting for geometrically nonlinear damage modelling framework. Computational Mechanics 2002;30:65-80. 
[14] Simo JC, Ju JW. Strain- and stress-based continuum damage models - I. Formulation. International Journal of Solids and Structures 1987;23:821-40. 